\title{
APPLICATION OF ARTIFICIAL NEURAL NETWORK AND GENETIC ALGORITHM TO HEALTHCARE WASTE PREDICTION
}

\author{
Samira Arabgol and Hoo Sang Ko \\ Department of Mechanical and Industrial Engineering, Southern Illinois University Edwardsville, \\ Edwardsville, IL 62026-1805, USA
}

\begin{abstract}
Prompt and proper management of healthcare waste is critical to minimize the negative impact on the environment. Improving the prediction accuracy of the healthcare waste generated in hospitals is essential and advantageous in effective waste management. This study aims at developing a model to predict the amount of healthcare waste. For this purpose, three models based on artificial neural network (ANN), multiple linear regression (MLR), and combination of ANN and genetic algorithm (ANN-GA) are applied to predict the waste of 50 hospitals in Iran. In order to improve the performance of ANN for prediction, GA is applied to find the optimal initial weights in the ANN. The performance of the three models is evaluated by mean squared errors. The obtained results have shown that GA has significant impact on optimizing initial weights and improving the performance of ANN.
\end{abstract}

\section{Introduction}

The number of healthcare facilities or medical establishment, such as hospitals, clinics, medical laboratories, pharmacies, and any support medical services, has been growing over the last decades. Consequently, there has been a significant increase, especially in developing countries, in generation of waste from the facilities. The total waste stream generated from these facilities is known as healthcare waste. Healthcare waste types vary based on their applications. For instance, World Health Organization (WHO) classifies healthcare waste into sharps, infectious, pathological, radioactive, and pharmaceuticals [1]. Mohamed et al. categorized hazardous healthcare waste as infectious, pathogenic, genotoxic, chemical, pharmaceutical, sharps, and waste with heavy metals content, pressurized containers and radioactive materials [2].

All individuals exposed to hazardous healthcare waste are potentially at risk, including those within healthcare establishments that generate hazardous waste, and those outside these sources who either handle such waste or are exposed to it as a consequence of careless management [3]. There is a particular concern about infection with human immunodeficiency virus (HIV) and hepatitis viruses B and $\mathrm{C}$, for which there is strong evidence of transmission via healthcare waste [4]. Among the different types of healthcare waste, sharps are critical since the viruses are generally transmitted through injuries from syringe needles contaminated by human blood [1]. In addition, infectious and anatomic wastes together account for up to $15 \%$ of the total waste from healthcare activities [5]. Therefore, these types of wastes have higher priority and thus should be managed and tracked to prevent diseases from spreading.

The actual amount of healthcare waste generated from healthcare facilities in the United States is difficult to estimate due to the inconsistency in the reported figures as well as the differences in the definition and tracking procedures for healthcare waste used by different groups. Moreover, the 
difficulty in determining the amount of home health care waste contributes to the uncertainty. However, some studies revealed a clear piece of evidence showing that the amount of hospital wastes has proliferated. For instance, the Environmental Protection Agency (EPA) reported the total generation rate of hospital wastes at 13 pound per bed per day in 1987, and another study in 1989 estimated hospital waste generation ranging between 16 to 23 pounds per bed per day [6]. These figures show that the amount of generation of healthcare wastes may have increased significantly within the decades. Healthcare wastes must be carefully tracked and managed to minimize their risks to environment and health; however, it is difficult to make an effective plan for effective handling of healthcare wastes due to the increase and uncertainty in healthcare waste generation.

Prediction methods can help achieve proper and prompt management of healthcare wastes by dealing with the uncertainty. Methods such as artificial neural networks (ANN) are used to estimate the healthcare waste generated by healthcare facilities [7]. ANN is one of the most common methods for prediction. Although ANNs are used for solving a variety of prediction problems [8], they have some restrictions. One of the most common is associated with neural network training. The learning algorithm, such as back-propagation algorithm, adjusts the network's weights and biases by calculating the gradient of the error. Usually, back-propagation neural networks are applied with random initial weight setting because of symmetry breaking. However, training the neural networks with random initial weights may cause two main issues: trapping into local minima and converging slowly [9]. As a result, the ANN is often unable to find a desirable solution. The performance of ANN can be improved by genetic algorithms (GA). GA are a class of global optimization techniques that mimics some of the processes observed in natural evolution [9]. GA can guide weight optimization which can quickly improve the performance of ANN [10].

This paper presents an ANN improved by a GA for accurate prediction of the amount of the healthcare waste in order to improve healthcare waste management. The remainder of the article is as follows. In Section 2, three intelligent techniques are applied to develop models for prediction of healthcare wastes. Section 3 illustrates numerical analyses to evaluate the performance of the models with real data. Finally, the study is concluded in Section 4.

\section{Methodology}

\subsection{ANN for prediction}

ANN can be defined as a model of reasoning based on the human brain. The brain consists of a densely interconnected set of basic informationprocessing units, called neurons. The neurons are connected by weighted links passing signals from one neuron to another. ANN learns through repeated adjustments of these weights.

A multilayer perceptron is a feedforward neural network with one or more hidden layers. Typically, the network consists of an input layer of source neurons, at least one middle or hidden layer of computational neurons, and an output layer of computational neurons. Feedforward networks are a class of ANN in which the information will be communicated only downstream in the network. There are different types of feedforward networks each of which applies a different technique to modify the weights for training. For example, the backpropagation neural network is an ANN that calculates the gradient of the error and then propagates error backward through the network to modify the weights and biases [11].

In order to improve the accuracy of healthcare waste prediction, an ANN model is developed with 6 input neurons, including four binary encoded inputs to indicate the healthcare facility type, one input for capacity, and one input for bed occupancy (more details provided in Section III). The ANN has a hidden layer with 10 neurons, and an output layer with one output neuron that yields the predicted amount of sharp wastes.

Figure 1 illustrates the ANN model. In ANN, weight values can be binary or real coded. Some studies used a binary representation of connection weights [12]. Binary weight can eliminate irrelevant values but it may cause significance loss. In the proposed ANN, real values are used for weights. There are total 70 weights on the ANN, including two sets of vectors: the first vector set containing 
weights between input and hidden layer and the second vector set for weights between hidden and output layer. The weights are initialized with random values between -1.0 to +1.0 .

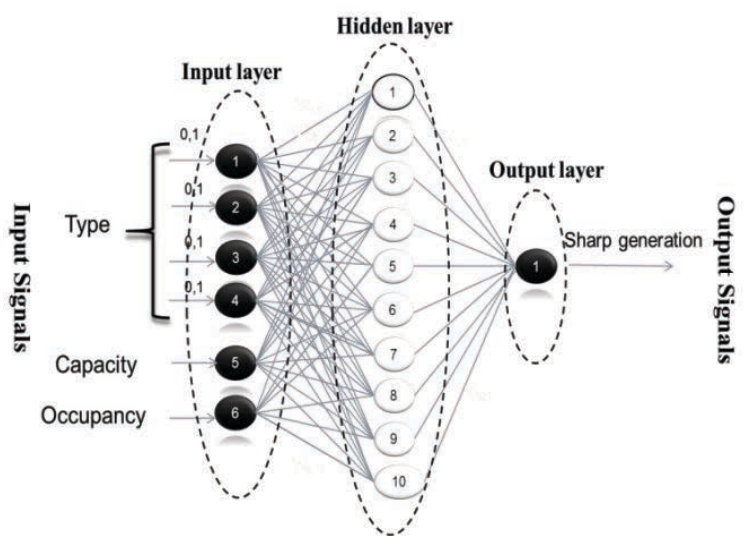

Figure 1. Healthcare waste prediction ANN model.

In the ANN, each neuron computes the net weighted input by:

$$
X=\sum_{i=1}^{n} x_{i} w_{i}-\theta
$$

where $x_{i}$ is the $i$-th input to the neuron, $w_{i}$ is the weight on the $i$-th link, $n$ is the number of inputs, and ? is the threshold applied to the neuron. Next, this input value is passed through the activation function:

$$
Y^{\text {sigmond }}=\frac{1}{1+e^{-x}}
$$

Then, the weights are changed through the supervised Multi-Layer Perceptron (MLP) feedforward training procedure based on a backpropagation algorithm. The available dataset will be divided into training, validation, and test datasets. After training the ANN, the performance of the ANN will be measured by mean squared error (MSE) for the test dataset [13].

Although the ANN should reduce MSE, there is no guarantee that the initial weights are efficiently selected, and the ANN may yield poor performance due to the initial weights. There have been studies of combining ANN and GA to optimize the learning process of ANN [14-16]. In this research, a GA is used for optimizing the selection of initial weights in order to improve the performance of the ANN for healthcare waste prediction.

\subsection{ANN augmented with GA}

GA are a class of stochastic search algorithms based on biological evolution, which generates solutions to optimization problems using techniques inspired by natural evolution, such as inheritance, mutation, selection, and crossover. In GA, a population of candidate solutions to an optimization problem is evolved toward better solutions. Each candidate solution has a set of properties, called chromosomes which can be altered and mutated to reproduce the next population with better fitness. The GA can be described by the following steps:

1 Choose the size of a chromosome, population, the crossover and mutation probabilities, and define the number of training epochs.

2 Define a fitness function to measure the performance.

3 Generate an initial population of chromosomes randomly.

4 Calculate the fitness function of each individual chromosome.

5 Select a pair of chromosomes for mating from the current population.

6 Create a pair of offspring chromosomes by applying the genetic operators - crossover and mutation, respectively.

7 Place the created offspring chromosomes in the new population.

8 Repeat Step 5 until the size of the new chromosome population becomes equal to the size of the initial population, $\mathrm{N}$.

9 Replace the initial (parent) chromosome population with the new (offspring) population.

10 Go to Step 4, and repeat the process until the termination criterion is satisfied.

A GA is applied to find an optimal set of initial weights for the multi-layer feedforward ANN. Initial weights in the network are chosen randomly within $[-1,1]$ [17]. A chromosome represents all the initial weights and biases in the ANN, which are optimized through the GA. The ANN has 70 weighted links (to be represented as genes in a chromosome) 
between neurons in the three layers, including: 1) an input layer with 6 neurons that represent type of hospital, hospital capacity, and bed occupancy; 2) a hidden layer with 10 neurons; and 3) an output layer with one output neuron. For this purpose, the GA randomly creates a population of 1000 chromosomes and selects 100 of them which yield better performance in the ANN (less MSE) using the roulette wheel selection method [18]. Roulette wheel selection is used for selecting potentially useful solutions for recombination. The advantage of roulette wheel selection is that it discards none of the individuals in the population and gives a chance to all of them to be selected. In addition, diversity in the population is preserved [19]. The probability for each individual being selected by roulette wheel selection can be calculated by:

$\mathrm{P}$ [Individual $i$ is chosen $]=\frac{F_{i}}{\sum_{j=1}^{n} F_{j}}$

where $F_{i}$ is the fitness of individual $i$ and $n$ is the population size. The chromosome's fitness is evaluated by the performance of the ANN (MSE). The relative fitness of the individual chromosome determines the chance of the chromosome being selected for mating.

Next, the GA applies crossover and mutation operations. Two-point crossover technique is used to reproduce new chromosomes from the selected for the next population. Then, a mutation operator is applied in order to maintain diversity within the population. Since the initial weights of the ANN (genes) could take any values between zero and one, the mutation is conducted by switching random genes. Finally, the best chromosome with the minimum MSE is chosen and set as the initial weights for the next period in the ANN [20]. Table 1 shows the ANN-GA parameters used in this study.

Figure 2 shows the overall ANN-GA process to optimize the initial weights of the ANN. At the first step, the initial population of individuals (chromosomes) is generated, each of which codifies a set of values for the weights of the connections and biases of the ANN. Then the fitness of each individual is evaluated, which entails allocating the values of the weights and biases codified by the individual to assess the network connections and calculating the mean square error. After training and testing the ANN, the error is calculated and used as the individual's fitness. After this initialization stage, the genetic selection, crossover and mutation operators are applied to reproduce the next population that will be used as the set of initial weights for the ANN. This step is repeated until there is convergence toward a population of individuals encoding the set of weights that keep the error at a desired level.

Table 1. ANN-GA parameters

\begin{tabular}{ll}
\hline Network parameters & Description \\
\hline Neural network architecture & $6-10-1$ \\
Transfer function & Sigmoid \\
Partitioning dataset & $70 \% / 15 \% / 15 \%$ \\
(Train/Valid/Test) & $(35 / 7 / 8)$ \\
Search method & GA \\
Population size & 100 \\
Fitness function & MSE \\
Probability of crossover & 0.85 \\
Probability of mutation & 0.019 \\
Crossover technique & Two point \\
Selection method & Roulette wheel \\
& selection \\
\hline
\end{tabular}

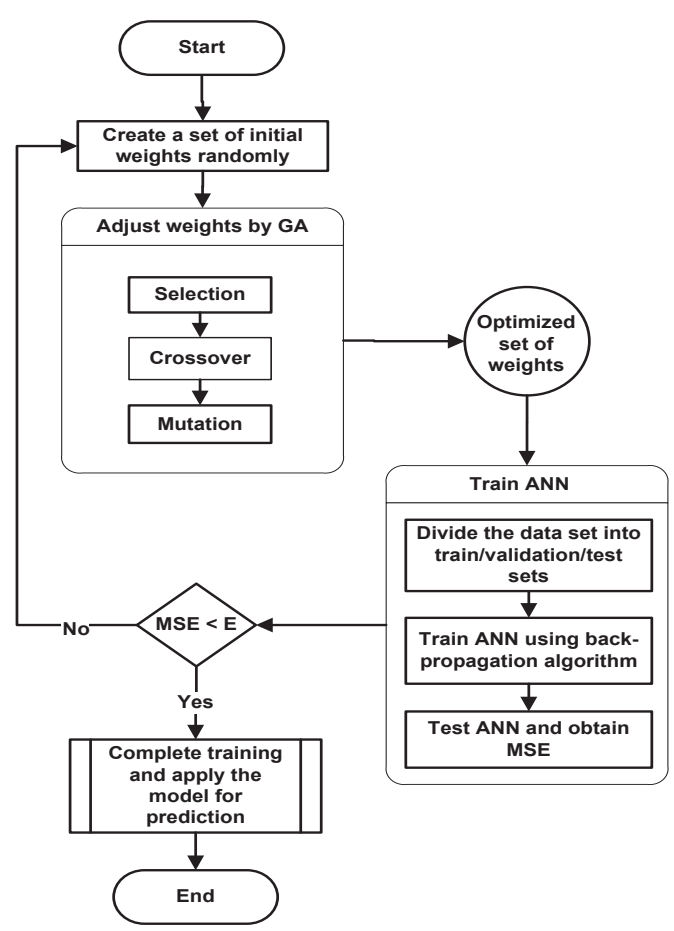

Figure 2. The ANN-GA algorithm 
In order to evaluate the results obtained from the ANN-GA, a multiple linear regression (MLR) model is developed on the same dataset for comparison.

\subsection{Multiple linear regression (MLR)}

In statistics, linear regression is an approach to model the relationship between a scalar dependent variable $y$ and one or more explanatory variables denoted $X$. There are two types of regression models: simple linear regression in which one input variable is used to estimate the response variable; and multiple linear regressions in which multiple explanatory variables are used to estimate the response variable.

If the goal is prediction, linear regression can be used to fit a predictive model to an observed data set of dependent variable $y$ and independent variables $x \in X$. After fitting a model, a new set of values for $X$ can be presented to the fitted model to make a prediction of the value of $y$. A MLR model for predicting the healthcare waste generation can be described as follows:

$$
y=\beta_{0}+\beta_{1} x_{1}+\beta_{2} x_{2}+\beta_{3} x_{3}+\cdots+\beta_{n} x_{n}
$$

The dependent variable $y$ represents the predicted value of generated waste, and $x_{1}, x_{2}, \ldots, x_{n}$ are independent variables used to predict response $y$. The coefficients $\beta_{0}, \beta_{1}, \ldots, \beta_{n}$ denote the impact of each input variables on response variable. In this example, $x_{1}, x_{2}$, and $x_{3}$ are dummy variables that are employed to identify the hospital types. Thus, no interaction exists between dummy variables and continuous variables, i.e. the impact of bed occupancy on healthcare waste does not change in different hospitals. However, the interaction between capacity and bed occupancy is considered in MLR. In total, there are five independent variables in the MLR as follows:

$$
\begin{gathered}
y= \\
=\beta_{0}+\beta_{1} x_{1}+\beta_{2} x_{2}+\beta_{3} x_{3}+\beta_{4} x_{4}+\beta_{5} x_{5}+\beta_{45} x_{4} x_{5}
\end{gathered}
$$

where $x_{i}=1$ if the waste type is $i(i=1,2,3), 0$ otherwise; $x_{4}$ is the capacity (number of beds); and $x_{5}$ is bed occupancy. The MLR will be used for comparison with the ANN-GA model.

\section{Evaluation}

This research presents an ANN with a GA developed to predict the amount of the sharps generated by healthcare facilities. A dataset, which includes 50 hospitals in Iran [21], has been used in order to train and test the ANN-GA model. The dataset includes three important parameters that may affect the waste generation: type of hospital, hospital capacity and bed occupancy. According to [21], hospital types were encoded as follows: private hospital $(1,0,0,0)$, governmental hospital $(0$, $1,0,0)$, educational hospital $(0,0,1,0)$ and noneducational, but university-related hospital $(0,0,0$, $1)$.

Three prediction methods are developed to improve healthcare waste estimations: ANN, ANNGA, and MLR that are applied to the aforementioned dataset. In order to develop these methods and test with the dataset, MATLAB Neural Network toolbox and Minitab are used. The ANN consists of an input layer with 6 neurons, a hidden layer with 10 neurons, and an output layer with one neuron. MSE is used to measure the performance of ANN.

The performance of ANN and ANN-GA is shown in Figs. 3 and 4, respectively. Figure 3 shows the training for the ANN stops at epoch 9; however, the test error starts increasing after epoch 6 while the training error keeps decreasing, which means that the model may overfit the training data. Figure 4 shows the three performance curves generated by the ANN-GA has similar trends, showing no sign of overfitting. The performance of the ANN-GA has been significantly improved over that of ANN as shown in Table 2.

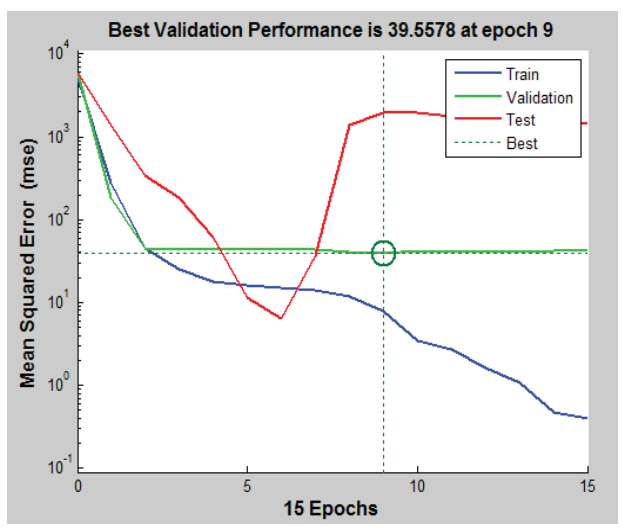

Figure 3. Performance of ANN 


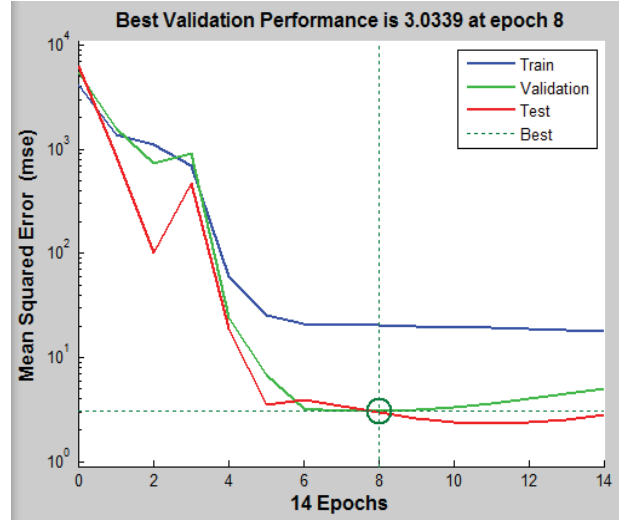

Figure 4. Performance of ANN-GA

Table 2. MSE of ANN, ANN-GA, and MLR

\begin{tabular}{ll}
\hline Model & MSE \\
\hline ANN & 1,930 \\
ANN-GA & 2.9563 \\
MLR & 28.8659 \\
\hline
\end{tabular}

For comparison, a MLR is developed to estimate the relationship between the healthcare waste and the three types input variables. Table 3 shows the coefficients of MLR parameters estimated using $70 \%$ of the dataset (training dataset). The fitted MLR model is shown in Equation (6):

$$
\begin{aligned}
& \text { Target }=-11.608+5.584 x_{1}-4.242 x_{2}+ \\
& 8.018 x_{3}+0.248 x_{4}-0.087 x_{5}-0.002 x_{45}
\end{aligned}
$$

Table 3. General regression analysis: coefficients

\begin{tabular}{lllll}
\hline & coef & $\begin{array}{l}\text { SE. } \\
\text { coef }\end{array}$ & t-value & p-value \\
\hline$\beta_{0}$ & -11.607 & 5.982 & -1.941 & 0.062 \\
$\beta_{1}$ & 5.584 & 4.766 & 1.172 & 0.251 \\
$\beta_{2}$ & -4.242 & 8.324 & -0.509 & 0.614 \\
$\beta_{3}$ & 8.018 & 4.577 & 1.752 & 0.091 \\
$\beta_{4}$ & 0.247 & 0.054 & 4.556 & 0.001 \\
$\beta_{5}$ & -0.0874 & 0.133 & -0.657 & 0.517 \\
$\beta_{45}$ & -0.002 & 0.001 & -2.515 & 0.018 \\
\hline
\end{tabular}

According to the p-value analysis in the general regression model shown in Table $3(\alpha=0.1)$, hospital type and capacity have significant effects on healthcare waste generation. In addition, since capacity and bed occupancy have interaction (small pvalue), bed occupancy is also included in the MLR model. The MSE for the MLR is 28.8569 .

Among the three methods applied to the dataset, ANN-GA method provides the smallest MSE (2.9563). The MSE of MLR (28.8659) is smaller than that of ANN (1.93E+03) but larger than that of ANN-GA. Thus, the results show that MLR is more accurate than random ANN to estimate the amount of healthcare wastes produced; however, by improving the initial weights using a GA, the accuracy of the ANN can be significantly improved, and thus it can reduce the uncertainty in healthcare waste generation. One of the reasons that the performance of the ANN is so poor is that the size of dataset is small with only 50 records. It is often the case because this kind of data is difficult to obtain. However, this study shows that the performance of ANN can be significantly improved by GA even though the volume of the dataset is not sufficient.

\section{Conclusion}

Accurate prediction of healthcare waste generation plays an important role in establishing a proper management plan on generation, collection, transportation, treatment and disposal of the healthcare waste. However, forecasting the exact amount of waste is challenging due to uncertainties in the healthcare environment such as the number of inpatients/outpatients per day, the type and number of surgery, and the technology level used in the hospital. To overcome these challenges and realize proper management of healthcare waste, this paper presents three methods to predict the amount of healthcare waste generated by healthcare facilities. The MLR model shows that hospital type, capacity, and bed occupancy are appropriate parameters to predict the healthcare wastes; however, the performance of the ANN based on those input parameters reveals that it is not an appropriate model to predict healthcare wastes, particularly when the available data set is limited. This paper presents an ANN model augmented by a GA to predict healthcare waste generation with high accuracy. By op- 
timizing the initial weights, the ANN-GA performs significantly better than other methods even though the size of the dataset is small [22]. The performance can be further improved by selecting suitable parameters in the GA such as selection criteria, crossover/mutation operators, and initial population. The difference between MSE of the ANN and that of the ANN-GA shows that setting appropriate initial weights are critical on the performance of ANN. In order to improve the model, the effect of other potential factors will be studied in the future with more extensive datasets. In addition, other prediction methods, such as bootstrapping and boosting, will be investigated in order to improve the accuracy of prediction.

\section{References}

[1] World Health Organization, Research for Universal Health Coverage, WHO Publications, 2013.

[2] L. F. Mohamed, S. A. Ebrahim, and A. A. AlThukair, Hazardous Halthcare Waste Management in the Kingdom of Bahrain, Waste Management, vol. 29, no. 8, 2009, pp. 2404-2409.

[3] W. Rutala, R. Odette, and G. Samsa, Management of Infectious Waste by US Hospitals, The Journal of the American Medical Association (JAMA), vol. 262, 1989, no. 12, pp 22-29.

[4] S. Altin, A. Altin, B. Elevil, and O. Cerit, "Determination of Hospital Waste Composition and Disposal Methods: a Case Study," Polish Journal of Environmental Studies, vol. 12, no. 2, 2003, p. 251-255.

[5] TERI Energy Data Directory and Yearbook 2007, The Energy and Resources Institute, 2007.

[6] U.S. Congress Office of Technology Assessment, Issues in Medical Waste Management, OTA publications, Washington, DC, 1988.

[7] H. Burke, P. Goodman, D. Rosen, D. Henson, J. Weinstein, F. Harrell, J. Marks, D. Winchester, and D. Bostwick, Arti?cial Neural Networks Improve the Accuracy of Cancer Survival Prediction, Cancer, vol. 79, no. 4, 1997, pp. 857-862.

[8] M. Adya and F. Collopy, How Effective are Neural Networks at Forecasting and Prediction, Journal of Forecasting, vol. 17, no. 5-6, 1998, pp. 481-495.

[9] Y. T. Chang, J. Lin, J. Shing Shieh, and M. F. Abbod, Optimization the Initial Weights of Artificial Neural Networks via Genetic Algorithm Ap- plied to Hip Bone Fracture Prediction, Advances in Fuzzy Systems, vol. 2012, 2012.

[10] M. Negnevitsky, Artificial Intelligence: A Guide to Intelligent Systems, 2nd Ed., Pearson Education, 2005.

[11] G. Li, H. Alnuweiri, Y. Wu, and H. Li, Acceleration of Back Propagation through Initial Weight Pre-training with Delta Rule, in: Proceedings of the IEEE International Conference on Neural Networks, vol. 1, pp. 580-585, San Fransisco, CA, 1993.

[12] S. Li, J. Yuan, X. Yue, and J. Luo, The BinaryWeights Neural Network for Robot Control, in: Proceedings of the 3rd IEEE RAS and EMBS International Conference on Biomedical Robotics and Biomechatronics, pp. 765-770, Tokyo, Japan, 2010.

[13] Y. Lee, S. H. Oh and M. W. Kim, The Effect of Initial Weights on Premature Saturation in Backpropagation Learning, in: Proceedings of the International Joint Conference on Neural Networks, vol. 1, 1991, pp. 765-770, Seattle, WA.

[14] R. S. Sexton and J. N. D. Gupta, Comparative Evaluation of Genetic Algorithm and Backpropagation for Training Neural Networks, Information Sciences, vol. 129, no. 1-4, 2000, pp. 45-59.

[15] D. J. Montana and L. Davis, Training Feedforward Neural Networks Using Genetic Algorithms, in: Proceedings of the 11th International Joint Conference on Artificial Intelligence, vol. 1, 1989, pp. 762-767.

[16] A. Kattan, R. Abdullah and R. A. Salam, Training Feed-Forward Neural Networks Using a Parallel Genetic Algorithm with the Best Must Survive Strategy, in: Proceedings of the International Conference on Intelligent Systems, Modelling and Simulation (ISMS), pp. 96-99, Liverpool, UK, 2010.

[17] J. A. Blackard and D. J. Dean, Comparative Accuracies of Arti?cial Neural Networks and Discriminant Analysis in Predicting Forest Cover Types from Cartographic Variables, Computers and Electronics in Agriculture, vol. 24, no. 3, 1999, pp. 131-151.

[18] T. D. Gwiazda, Genetic Algorithms Reference: Crossover for Single Objective Numerical Optimization Problems, Lightning Source Inc., 2007.

[19] N. M. Razali and J. Geraghty, Genetic Algorithm Performance with Different Selection Strategies in Solving TSP, in: Proceedings of the World Congress on Engineering, vol. 2, London, UK, 2011. 
[20] D. E. Goldberg, Genetic Algorithms in Search, Optimization, and Machine Learning, AddisonWesley, 1989.

[21] S. Jahandideh, S. Jahandideh, E. Barzegari Asadabadi, M. Askarian, M. M. Movahedi, S. Hosseini, and M. Jahandideh, "The Use of Artificial Neural Networks and Multiple Linear Regression to Predict Rate of Medical Waste Generation,"
Waste Management, vol. 29, no. 11, 2009, pp. 2874-2879,.

[22] D. Venkatesan, K. Kannan, and R. Saravanan, ”A Genetic Algorithm-based Artificial Neural Network Model for the Optimization of Machining Processes," Neural Computing and Applications, vol. 18, no. 2, 2009, pp. 135-140. 